\title{
A Recent Clinical Update on Epilepsy in Pregnancy
}

\author{
Isabel Arroyo, $\mathrm{BHSc}^{1}$ \\ ${ }^{1}$ Faculty of Medicine, University of Ottawa
}

A B STRACT

Chronic diseases can have numerous effects across a person's lifespan. Epilepsy is a chronic disease that impacts all stages of life including pregnancy. Pregnancy is a sensitive time in one's life, especially for people living with a chronic disease. This review presents a recent update on the scientific findings surrounding epilepsy and pregnancy. Topics such as conception, seizure control during pregnancy, health risks to the mother and baby during pregnancy and side effects of anti-epileptic medications will be covered. Through the presentation of findings from 2013 to 2015, this review will update future clinicians on novel developments in the field of pregnancy and epilepsy that will aid with treatment of patients, while simultaneously identifying gaps in the literature and needs for future research. This review serves as a reminder that medicine needs to be tailored to the unique individual and that individuals living with chronic diseases may require altered treatment plans throughout their lives.

\section{RÉSUMÉ}

Les maladies chroniques peuvent avoir de nombreux effets au travers de la vie d'une personne. L'épilepsie est une maladie chronique qui touche tous les stades de la vie, y compris la grossesse. La grossesse est un moment délicat de la vie, en particulier pour les personnes vivant avec une maladie chronique. Cette revue présente une mise à jour récente sur les découvertes scientifiques au sujet de l'épilepsie et de la grossesse. Des sujets tels que la conception, le contrôle des crises pendant la grossesse, les risques pour la santé de la mère et du bébé pendant la grossesse et les effets secondaires des médicaments antiépileptiques seront couverts. Par le biais de la présentation des résultats de 2013 à 2015, cette revue servira de mise à jour des nouveaux développements dans le domaine de la grossesse et de l'épilepsie pour les futurs cliniciens et aidera avec le traitement des patients, tout en identifiant simultanément les lacunes dans la littérature et les besoins pour la recherche future. Cet examen sert comme un rappel que la médecine doit être adaptée à l'individu unique et que les personnes atteintes de maladies chroniques peuvent exiger des plans de traitement modifiés tout au long de leur vie.

\section{INTRODUCTION}

Chronic disease can affect different facets of one's life. For instance, women living with chronic diseases face particular medical risks and challenges during pregnancy. Epilepsy is defined as the occurrence of a seizure in an individual with a high likelihood of reoccurrence, two or more recurrent seizures, or a syndrome involving seizures $[2,3]$. Epilepsy is the most common neurological disorder in pregnant women [1]. One and a half million women of childbearing age have epilepsy. Three to five births per 1000 are to mothers with epilepsy [2]. The challenge in the treatment of epilepsy during pregnancy is finding a balance between the detrimental effects of having uncontrolled seizures and the teratogenic side effects of seizure controlling medications on the fetus.

This review aims to present advances in the literature from 2013 to 2015 surrounding epilepsy and pregnancy, while identifying gaps in knowledge that require future research. Updates on important clinical topics involved in the treatment of women with epilepsy will be discussed. Subsequently, topics such as conception, seizure control during pregnancy, health risks to the mother and baby during pregnancy, and side effects of anticonvulsant medications will be covered. This review serves as a reminder that medicine needs to be altered for the individual in the context of comorbid disease.

\section{CITATION SELECTION CRITERIA}

A literature search using the University of Ottawa's library search engine was performed. An advanced search was employed using the keywords "epilepsy" and "pregnancy." The search was restricted to studies that were published from 2013 to 2015, written in English, and peer-reviewed. Cohort studies, case-control studies and clinical trials were all included. The subject tool was then applied, and the subject of "depression" was excluded

Keywords: Epilepsy; Pregnancy; Risks; Teratogen 


\section{Review and Clinical Practice}

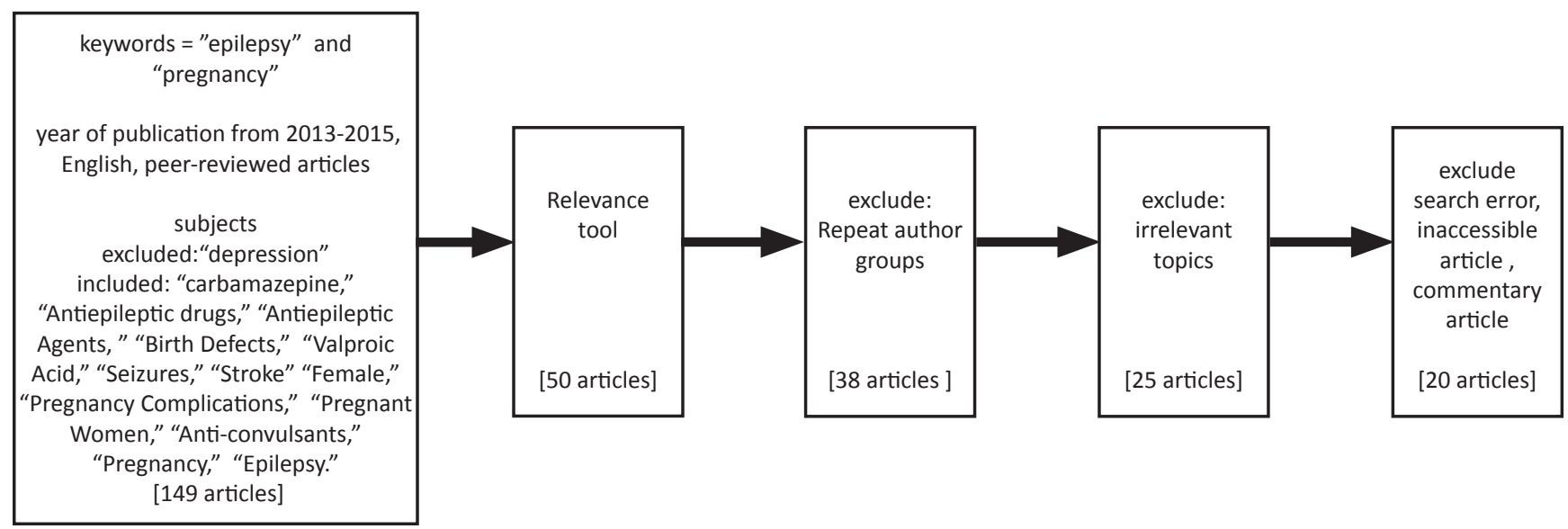

Figure 1. Methodology Inclusion and Exclusion Criteria

Note: Three articles were discarded because they did not fit the publication year or language criteria (search error)

because results including depression focused on the topic of postpartum depression. The following subjects were included: "Carbamazepine", "Antiepileptic drugs", "Antiepileptic Agents", "Birth Defects", "Valproic Acid", "Seizures", "Stroke", "Female", "Pregnancy Complications", "Pregnant Women", "Anticonvulsants", "Pregnancy" and "Epilepsy." This search strategy yielded 149 articles. The articles were sorted by relevance, and the 50 most relevant articles were included (the relevance of subsequent results declined greatly). In order to eliminate possible overrepresentation of one group of researchers, multiple articles written by the same author or group of authors were excluded. By eliminating repeated authors, the total number of articles was reduced to 38.13 articles discussing the following subjects were deemed irrelevant by the author: specific nursing training initiatives, isolated animal studies, use of antiepileptic drugs in the setting of psychiatric illness, pregnancy risk factors for childhood epilepsy, neuroprotective effects of perinatal choline nutrition and pregnancy risk factors not associated with epilepsy. Two articles were excluded because they were erroneously included by the automatic search (one was not in English and the other was published in 2004). Additionally, the full text of one article could not be accessed online and was thus excluded. Lastly, one article was written in response to another article and was also excluded. The final number of articles included in the review was 20, as illustrated in the following flow chart (Figure 1).

\section{CONCEPTION \& FERTILITY}

Many women with epilepsy have unique medical problems that may make conception difficult. A third of women with epilepsy are infertile, which is defined as lack of successful conception after a one-year period of unprotected sexual activity. Thomas et al. studied women who were either trying to conceive or in early pregnancy and followed them until their children were 12 years old [3]. It was found that the infertile women with epilepsy had higher levels of luteinizing hormone than dehydroepiandrostene- dione, as well as lower levels of progesterone than women with epilepsy who were fertile [3]. The differences in hormone levels and association between epilepsy and infertility is hypothesized to be mediated by the fact that epilepsy is an endocrine disruptor, which can result in hypothalamic disruption and increased androgen levels in females. This effect on fertility is compounded by secondary outcomes of anti-epileptic drugs [3]. Therefore, patients with epilepsy should be supplied with additional counsel and treatment pertaining to infertility.

On the other hand, it is interesting that certain anti-epileptic medications have been hypothesized to interfere with the efficacy of birth control pills [1]. Medication interference with the oral contraceptive pill should be discussed with all female patients on antiepileptic medications. One study found that $26 \%$ of pregnancies in women with epilepsy result from contraception failure [1].

\section{EFFECTS OF PREGNANCY ON SEIZURE CONTROL}

Monitoring and controlling seizure frequency is important during pregnancy. Having one or more generalized tonic-clonic seizures during pregnancy is associated with a higher risk of preterm birth [4]. A recent study found that $19 \%$ of women experience an over $50 \%$ increase in seizure frequency during pregnancy, $8 \%$ experience a decrease in seizure frequency of $50 \%$ or more, and $72 \%$ have no change. The findings of this study are limited by a small sample size (36 cases and 72 controls); larger scale studies are required to corroborate these results [5].

Another important area of research is the efficacy of seizure control medications in pregnant individuals. Deterioration in seizure control during pregnancy is associated with physiological changes in the maternal body that result in increased drug clearance. For example, a study comparing monotherapy with carbamazepine, lamotrigine, phenobarbital and valproate used in pregnant women concluded that women using lamotrigine 
had more generalized tonic-clonic seizures, were more likely to deteriorate in seizure control from their first trimester to their last trimester, and were more likely to require an increase in dose throughout their pregnancy [6]. However, this study is limited by the fact that randomized controls were not used and preliminary data on seizure control was not collected [6]. In general, studies on this topic are also limited by small sample sizes as well as regulations restricting the allowable volume of blood drawn from pregnant women [7].

\section{RISK TO MOTHER}

The risk of death during pregnancy for women with epilepsy is estimated to be ten times higher than in women without epilepsy [8]. A study analyzing data from the United Kingdom Confidential Enquiries into Maternal Deaths (UKCEMD), a report that collected data on all pregnancies in the United Kingdom from 2006 to 2008, found that 14 out of an estimated 13,978 pregnant women with epilepsy died from epilepsy-related complications during or shortly after pregnancy [8]. Of these 14 deaths, 11 were sudden and unexpected [8]. Sudden and unexpected death in epilepsy (SUDEP) is defined as death in a patient with epilepsy, excluding documented status epilepticus cases, where an autopsy has been performed to rule out trauma, drowning, and toxicological or anatomical causes of death [8]. Of the 14 deaths, 9 women were taking lamotrigine; the authors speculate that the high proportion of deaths in women taking lamotrigine reflects either an involvement of lamotrigine in SUDEP or physicians' preferences to prescribe lamotrigine in the United Kingdom [8]. One caveat of this study, which analyzed data from the UKCEMD report, is that the UKCEMD report did not record the number of women who had epilepsy [8]. Thus, the authors Edey et al. estimated the number of women who had epilepsy using a previously reported prevalence rate of epilepsy during pregnancy in the United Kingdom [8]. Evidently, more studies investigating the safety of lamotrigine use during pregnancy are needed.

\section{RISK TO BABY}

Authors Campbell, Devenney and Morrow observed that women with epilepsy who have had a previous child with congenital malformations have a $16.9 \%$ risk of having another child with congenital malformations [9]. In comparison, women with epilepsy who have not had a child with congenital malformations had a $9.8 \%$ risk of having a subsequent child with congenital malformations [9]. Although more studies are needed to confirm this phenomenon, these findings may suggest that genetics play a role in modulating the teratogenic effects of anti-epileptic medications [9]. On the other hand, a study analyzing pregnancy data from the Kerala Registry of Epilepsy and Pregnancy found that there is no increased risk of reoccurrence of congenital malformations in subsequent pregnancies: of those who had two pregnancies, 21 of 246 (8.5\%) women had major congenital malformations in the first pregnancy and 22 of 246 (8.9\%) had major congenital malformations in the second pregnancy. These numbers are comparable to the $7.2 \%$ rate of major congenital malformations in the children of women who had one recorded pregnancy [10].

It has also been observed that compared to the general population, women taking one anti-epileptic drug have twice as high a risk of giving birth to a baby that is small for gestational age, for their baby to have an Apgar score of less than seven (usually requires medical attention) at one minute after birth, or experience a spontaneous abortion [2]. Interestingly, another study showed that the increased risk of preterm delivery is only seen in mothers with epilepsy who are also smokers [2]. Contradictorily, a retrospective cohort study of 440 pregnant women with a self-reported seizure disorder found that women are not at an increased risk for intrauterine growth restriction, stillbirth, preeclampsia or premature delivery [11]. The perinatal risks of epilepsy seem to be unclear, and more data are required.

\section{COMPARING TERATOGENIC EFFECTS OF MEDICATIONS}

Up-to-date knowledge regarding the teratogenic effects of antiepileptic medications is imperative in the treatment of women with epilepsy. Each year in the United States, 7900000 women aged 15 to 44 receive prescriptions for anti-epileptic drugs [12]. A study with a cohort of pregnant women with epilepsy taking anti-epileptic drugs, a cohort of epileptic pregnant women not taking any drugs, and a cohort of non-epileptic pregnant women showed that the rate of major congenital malformations was only increased significantly in the epileptic group taking medications [13]. The rate of major congenital malformation in the general population is anywhere between 2 and 4 percent [14]. In a prospective cohort study, Veiby et al. found that children who were exposed to anti-epileptic drugs in utero have an increased risk of abnormal gross motor skills and autistic traits at 18 months of age, with odds ratios of 2.0 and 2.7 respectively [15]. A study of 1290 pregnant women prescribed a regimen of valproic monotherapy found that valproic acid is associated with a $6.7 \%$ risk of major congenital malformations [16]. The use of valproic acid is associated with neural tube defects, reduced verbal abilities, oral-facial clefts, hypospadias, congenital heart defects and skeletal abnormalities [2, 17]. Furthermore, a population-based study in Denmark found that valproic acid use during pregnancy significantly increases the risk of autism spectrum disorders in offspring. The study included 508 children who were exposed to valproic acid in utero. This risk remained significant when the authors controlled for parental psychiatric conditions, epilepsy and comorbid major congenital malformations. When mothers who had previously been on valproic acid discontinued the medication for at least 30 days prior to conception, the risk of autism was found to be equivalent to that of the general population. The 
implications of this study by Christensen et al. is limited because they did not control for folate supplementation or alcohol and illicit drug use during the course of the pregnancies [18].

Although the previous studies mentioned above found lamotrigine's control of seizures in pregnancy to be questionable, a study of 4000 pregnancies found that lamotrigine dosed at $300 \mathrm{mg}$ or less results in the lowest risk of congenital malformations [19]. The most common malformation associated with lamotrigine is cleft lip. $2.3 \%$ of a sample of 2198 women on lamotrigine gave birth to babies with major congenital malformations. The same study found higher risk rates associated with carbamazepine and valproic acid (2.6 and 6.7 respectively) [16].

The incidence rate of major congenital malformations with carbamazepine monotherapy is $2.6 \%$, which falls between those of valproic acid and lamotrigine [16]. In addition, the International Registry of Antiepileptic Drugs and Pregnancy Study found that low dose carbamazepine has a $3 \%$ risk of congenital malformations while the risk is $5 \%$ and $8.7 \%$ for medium and high doses respectively. [7].

Currently, newer drugs such as levetiracetam, oxcarbazepine and gabapentin are being used during pregnancy. Of 304 women who were exposed to monotherapy of levetiracetam, 2 women gave birth to children with major congenital malformations $(0.70 \%$; $95 \%$ confidence interval $0.19 \%-2.51 \%$ ) [20]. Of 248 pregnant women taking a monotherapy of oxacarbazepine, 6 women gave birth to offspring with a major congenital malformation [14]. The rate of birth defects with gabapentin use is uncertain with reported values ranging from $0 \%$ to $6 \%$ [2]. A study of 223 pregnant women exposed to gabapentin and 223 pregnant controls demonstrated that the rate of major congenital malformation in the two groups was the same [21]. As mentioned before, it is important to stress that when compared to the risk of major congenital malformations in the general population (2-4\%), the risks of major congenital malformation with lamotrigine, carbamazepine, levetiracetam, oxcarbazepine and gabapentin are not increased [14].

\section{TREATMENT GUIDELINES}

There are general treatment guidelines for epileptic control during pregnancy, but individually tailored treatment regimens may be necessary. First, women taking anticonvulsant medications should take folate to prevent neural tube defects. Although there is some controversy surrounding the recommended folate doses, the American College of Obstetricians and Gynecologists recommends that pregnant women taking anticonvulsants should take $4.0 \mathrm{mg}$ of folic acid per day [2]. If a woman has been seizure-free for a year, one can consider removing all anticonvulsant medications during pregnancy [2]. If the patient has not been seizure-free for a year, the ideal treatment during pregnancy is monotherapy with a drug of relatively low teratogenicity at the lowest effective dose for that individual [2]. The medication dose may need to be increased during pregnancy to account for the increase in plasma volume, cardiac output, renal blood flow along with the decrease in albumin concentration, a drug-binding protein. Also, pregnant women have increased activity of cytochrome P450 enzymes; therefore, drug metabolism will increase. In particular, lamotrigine is thought to have increased clearance during pregnancy [7]. Medications should be continued throughout delivery and breastfeeding, but doses need to be readjusted after pregnancy to account for changes back to physiological baseline [2]. In terms of drug selection, the drugs of choice during pregnancy are levetiracetam, oxcarbazepine and gabapentin [22]. Valproic acid should be avoided in pregnancy as it has the highest rate of major congenital malformations [2]. A retrospective cohort study of 153 women that compared a group of women undergoing planned pregnancies versus a group of women undergoing unplanned pregnancies showed that the planned pregnancies group had more women on monotherapy ( $80 \%$ planned vs. $61 \%$ unplanned). Also, more women in the planned pregnancy group were not on valproic acid with $77 \%$ in the planned group not on valproic acid versus $56 \%$ of the unplanned pregnancy group [23]. Thus, planned pregnancies are safer than unexpected pregnancies in women with epilepsy as women often do not realize they are pregnant within the first trimester, a time when the fetus is most sensitive to teratogens [23].

\section{CONCLUSION}

Epilepsy during pregnancy is a complex medical issue to treat. The most recent research on epilepsy during pregnancy must guide treatment selection for these patients. Epilepsy can lead to problems from conception to the postpartum period. Clinicians must be vigilant about seizure control and choice of medication during pregnancy. Evidence supports the fact that valproic acid is associated with an increased risk of congenital malformations. This review presents studies published from 2013 to 2015 with the aim of updating clinicians on recent findings in this field. There are still many questions surrounding best evidence based practices. Overall, this review serves as a reminder that medicine needs to be tailored to the individual patient, unique as she is.

\section{REFERENCES}

1. Ruth D, \& Barnett J. Epilepsy in Pregnancy: Best Evidence for Care. Journal of Perinatal \& Neonatal Nursing. 2013;27(3): 217.

2. Hart LA, \& Sibai, BM. Seizures in pregnancy: Epilepsy, eclampsia, and stroke. Seminars in Perinatology. 2013;37(4):207-224.

3. Thomas S, Sarma P, Nirmala C, Mathai A, Thomas A. Women with Epilepsy and Infertility Have Different Reproductive Hormone Profile than Others. Annals of Indian Academy of Neurology. 2013;16(4):544.

4. Rauchenzauner $M$, Ehrensberger M, Prieschl M, et al. Generalized tonic clonic seizures and antiepileptic drugs during pregnancy--a matter of importance for the baby? Journal of Neurology. 2013;260(2):484-8.

5. Neve A, Boero G, Francavilla T, Plantamura M, Agazio G, Specchio L. Pro- 
spective, case-control study on the effect of pregnancy on seizure frequency in women with epilepsy. Neurological Sciences. 2015;36(1):79-83.

6. Battino D, Tomson T, Bonizzoni E, et al. Seizure control and treatment changes in pregnancy: Observations from the EURAP epilepsy pregnancy registry. Epilepsia. 2013;54(9):1621-1627.

7. Johnson EL, Stowe ZN, Ritchie JC, et al. Carbamazepine clearance and seizure stability during pregnancy. Epilepsy \& Behavior, 2014;33:49-53.

8. Edey S, Moran N, Nashef L. SUDEP and epilepsy-related mortality in pregnancy. Epilepsia, 2014;55(7):e72-e74.

9. Campbell E, Devenney E, Morrow J,et al. Recurrence risk of congenital malformations in infants exposed to antiepileptic drugs in utero. Epilepsia. 2013; 54(1):165-171.

10. Begum S, Sarma $\mathrm{S}$, Thomas $\mathrm{S}$. Malformation in index pregnancy in women with epilepsy is not followed by recurrence in subsequent pregnancy. Epilepsia. 2013;54(12): E163-E167.

11. McPherson JA, Harper LM, Odibo AO, Roehl KA, Cahill AG. Maternal seizure disorder and risk of adverse pregnancy outcomes. American Journal of $\mathrm{Ob}$ stetrics and Gynecology. 2013; 208(5):378.e1-378.e5.

12. Meador KJ, Loring DW. Risks of In Utero Exposure to Valproate. JAMA. 2013;309(16):1730-1731.

13. Cassina M, Dilaghi A, Di Gianantonio E, et al. Pregnancy outcome in women exposed to antiepileptic drugs: Teratogenic role of maternal epilepsy and its pharmacologic treatment. Reproductive Toxicology. 2013;39: 50-57.

14. Montouris G. Safety of the newer antiepileptic drug oxcarbazepine during pregnancy. Current Medical Research and Opinion. 2005;21(5):693-701.

15. Veiby G, Daltveit AK, Schjølberg $S$ et al. Exposure to antiepileptic drugs in utero and child development: A prospective population-based study. Epilepsia. 2013;54(8):1462-1472.

16. Morrow J. Malformation Risks of antiepileptic drug monotherapies in pregnancy: updated results from the UK and Ireland epilepsy and pregnancy registers. Journal of Neurology, Neurosurgery \& Psychiatry. 2014;85(9):1029.

17. Koo J, Zavras A. Antiepileptic drugs (AEDs) during pregnancy and risk of congenital jaw and oral malformation. Oral Diseases. 2013;19(7):712-720.

18. Christensen J, Gronborg T K, Sorensen M J et al. Prenatal valproate exposure and risk of autism spectrum disorders and childhood autism. The Journal of the American Medical Association. 2013;309(16):1696.

19. Vajda, FJ, Seetal D, Horgan D. Lamotrigine in Epilepsy, Pregnancy and Psychiatry - a Drug for all Seasons? Journal of Clinical Neuroscience. 2013;20(1):13-6.

20. Mawhinney E, Craig J, Morrow J et al. Levetiracetam in pregnancy: Results from the UK and Ireland epilepsy and pregnancy registers. Neurology. 2013;80(4):400-5.

21. Fujii $\mathrm{H}$, Goel A, Bernard N et al. Pregnancy outcomes following gabapentin use: Results of a prospective comparative cohort study. Neurology. 2013;80(17):1565-70.

22. Eadie M. Treating epilepsy in pregnant women. Expert Opinion on Pharmacotherapy. 2014;15(6):841-850.

23. Abe K, Hamada H, Yamada T, Obata-Yasuoka M, Minakami H, Yoshikawa H. Impact of planning of pregnancy in women with epilepsy on seizure control during pregnancy and on maternal and neonatal outcomes. Seizure: European Journal of Epilepsy. 2013;23(2):112-116. 